\title{
La sociedad civil en Ecuador como agente del desarrollo: aportes y desafíos actuales
}

Civil Society in Ecuador as an Agent of Current Development, Contributions and Challenges

A sociedade civil no Equador como agente do desenvolvimento atual, contribuições e desafios

\section{Guido Cueva}

Pontificia Universidad Católica del Ecuador (Quito, Ecuador)

benji_gui@hotmail.com

DOI: https://doi.org/10.32719/25506641.2018.4.2

Fecha de presentación: 13 de abril de 2018 • Fecha de aceptación: 14 de septiembre de 2018 Artículo de investigación

Licencia Creative Commons

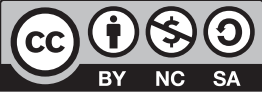




\section{Resumen}

Las organizaciones de la sociedad civil (OSC) en Ecuador desde su origen han determinado su compromiso dentro de la sociedad asegurar desde la sobrevivencia, reproducción y calidad de vida del ser humano hasta desarrollar líneas de trabajo concretas como derechos, política pública, ambiente entre otras. Sin embargo, desde el año 2007 se distingue un entorno de tensión entre el ejercicio del poder político con las organizaciones sociales y sus miembros, por prevalecer dentro de la esfera pública los intereses políticos del gobierno que no concordaron con la realidad y contexto de la población. El presente artículo busca describir y evaluar los aportes y desafíos de las OSC como agentes del desarrollo durante la presidencia de Rafael Correa, es decir, el contexto en el que se han desenvuelto, las fortalezas y conflictos que mantienen para el ejercicio diario de su trabajo como actores del desarrollo de nuestro país.

Palabras clave: sociedad civil, participación, Revolución Ciudadana.

JEL: M14 Cultura corporativa; responsabilidad social corporativa; O1 Desarrollo económico.

\section{Abstract}

Social organizations in Ecuador have determined their commitment within society to ensure the survival, reproduction and quality of life of human beings to develop specific lines of work. However, in 2007 there is an atmosphere of tension between those who exercise political power and organizations. This article describes and evaluates the contributions and challenges of the CSOs during the presidency of Rafael Correa, that is the context in which they have developed, the strengths and conflicts that they maintain for the daily exercise of their work as actors of the development of our country.

Keywords: civil society, participation, citizen revolution.

JEL: M14 Corporate culture; Corporate social responsibility; O1 Economic development.

\section{Resumo}

Organizações sociais no Equador determinaram seu compromisso na sociedade para garantir a sobrevivência, reprodução e qualidade de vida dos seres humanos para desenvolver linhas específicas de trabalho. No entanto, em 2007, existe uma atmosfera de tensão entre aqueles que exercem o poder político e as organizações. Este artigo descreve e avalia as contribuições e desafios das OSC durante a presidência de Rafael Correa, ou seja, o contexto em que se desenvolveram, os pontos fortes e os conflitos que eles mantêm para o exercício diário de seu trabalho como atores do desenvolvimento de nosso país.

Palavras-chave: sociedade civil, participação, Revolución Ciudadana.

JEL: M14 Cultura corporative; responsabilidade social corporative; O1 Desenvolvimento Econômico. 


\section{Introducción}

D or medio del presente trabajo de investigación se pretende identificar cuáles han sido los aportes y desafíos de las organizaciones de la socie-

1 dad civil como agentes del desarrollo en Ecuador, es decir, el contexto en el que se han desenvuelto y las fortalezas y conflictos que mantienen para el ejercicio diario de su trabajo como actores del desarrollo del país.

Es por ello, que surge el interés tanto académico como personal de identificar y conocer de forma histórica y conceptual las razones por las que los individuos se organizan dentro de la sociedad, así como conocer los aportes de distintas vertientes teóricas que permiten, desde el contexto ecuatoriano, identificar el trabajo de las organizaciones de la sociedad civil como promotoras del desarrollo.

El método para desarrollar esta investigación se desenvuelve en el abordaje del tema de las organizaciones de la sociedad civil mediante la recopilación, análisis y selección de información cualitativa en forma documental. Debido a que la investigación proyecta conceptos como Estado, organización, sociedad, desarrollo y participación, se hace necesario afrontar dicha problemática mediante un tipo de investigación teórica en donde el análisis del tema incluya una serie de conceptos surgidos por diferentes lineamientos teóricos que enmarcan una problemática dentro de un contexto teórico específico.

A continuación, se establece el desarrollo del concepto de sociedad civil desde distintas perspectivas teóricas bajo la línea del tiempo como medida establecida por el autor con el objetivo de contar con distintas aproximaciones desde el campo conceptual para establecer el origen y desarrollo tanto del concepto como de su propia materialización en Ecuador.

Se denominan organizaciones de la sociedad civil (OSC), a los colectivos que representan a los ciudadanos en la esfera pública, espacio que lo 
conforma el Estado y el sector privado. Uno de los primeros acercamientos al concepto de sociedad civil surge desde Aristóteles, al nombrar al grupo de ciudadanos que comparten un mismo valor y que buscan como fin último la felicidad, concluyendo que la sociedad civil es el espacio intermedio entre el Estado y la sociedad. Desde el pensamiento clásico hasta autores del siglo XXI, la sociedad civil es analizada como el sitio adecuado donde los valores morales, éticos y políticos se comparten, los sujetos conforman entre ellos lugares concretos para lograr sus objetivos en base a sus valores y participación activa en el escenario social.

Las organizaciones sociales surgen en la sociedad ya que el ser humano no puede asegurar desde sus limitaciones naturales la sobrevivencia por sí solo, al mismo tiempo que el sujeto en su proceso social de reproducción genera distintos elementos que tienen por objetivo asegurar la calidad de vida. La presencia del Estado responde a la necesidad de asegurar la existencia del ser humano y, al no responder a los intereses colectivos, son las organizaciones sociales aquellas que en un primer momento demandan o reclaman la razón de ser de un Estado. Al pasar de la demanda a la protesta en un segundo momento, las organizaciones sociales generan la lucha social, que se convierte en el motor de transformación de la sociedad al mantener como principio y en todo momento la sobrevivencia y calidad de vida del ser humano.

El reconocer que existen espacios donde las personas pueden organizarse en la sociedad, y que estos mismos han surgido como consecuencia de la lucha social a lo largo de la historia, es evidencia suficiente para reconocer el acercamiento y disposición que se pretende del ciudadano con el Estado. Por tal motivo, en un primer momento se dispone a realizar una breve reseña sobre el concepto, origen e importancia de las organizaciones sociales desde lo que denominamos la "sociedad moderna".

No existe bajo ninguna línea del tiempo una fecha u origen que defina el punto de partida de la creación de las organizaciones sociales. Por lo tanto, para el presente análisis es indispensable nombrar ciertos eventos en la historia que demuestran indudables vestigios de lo que la ciencia social deno-

1. Se denomina sociedad moderna al proceso en el cual se define la eficacia de la racionalidad instrumental en conjunto con el surgimiento del sujeto humano como libertad y creación (Touraine 2000). 
mina sociedad civil. Desde el pensamiento de Aristóteles, se menciona a la sociedad civil, en su texto de la Política, como una asociación de individuos caracterizados por mantenerse unidos de forma libre e igualitaria, siempre y cuando se encuentren inmersos en la polis. ${ }^{2}$

Aquella premisa bajo su pensamiento establece un vínculo entre los sujetos, guiados por el ideal del bien común y la superposición del interés general. Sin embargo, para la existencia de este concepto es necesario, menciona Aristóteles (capítulo V), contar con una aclaración de los tipos de gobierno. Por lo que establece la diferencia entre el gobierno que nace por la asociación de distintos y se legitima en cuanto busca el interés general, y aquel que nace con el único objetivo del interés particular.

Todo Estado es, evidentemente, una asociación, y toda asociación no se forma sino en vista de algún bien, puesto que los hombres, cualesquiera que ellos sean, nunca hacen nada sino en vista de lo que les parece ser bueno. Es claro, por tanto, que todas las asociaciones tienden a un bien de cierta especie, y que el más importante de todos los bienes debe ser el objeto de la más importante de las asociaciones, de aquella que encierra todas las demás, y a la cual se llama precisamente Estado y asociación política (Aristóteles, 1962, 1).

El Estado como ideal en el cual se legitima la búsqueda del bien común desde la perspectiva filosófica de Aristóteles, engloba a las asociaciones y o grupos de personas que han de prevalecer los intereses comunes frente a aquellos que demuestran los modelos viciosos de otro tipo de Estado. Y esto es, pues, resultado de lo que él mismo menciona como areté, ${ }^{3}$ definición griega que engloba la virtud, pues es conocido que para Aristóteles el fin último de la vida es la felicidad.

En segundo lugar, en la historia antigua en Roma bajo la existencia de un Estado se denomina, desde el análisis de Borja (2011), societas civilis como aquella forma de representación ciudadana guiada en principio por la inconformidad del ciudadano con su gobierno, esta era legítima en cuanto se garantizaba bajo la ley.

2. En la antigua Grecia, Estado autónomo constituido por una ciudad y un pequeño territorio (Real Academia de la Lengua Española, versión digital 2017).

3. Para que haya felicidad completa debe haber vida completa de areté (Aristóteles 1962). 
Sobre la premisa filosófica, Aristóteles deja por sentado una base de lo que es sociedad civil; sin embargo, dentro de la discusión es importante mencionar que dichos principios no definían aún lo que se denomina como sociedad política y sociedad civil. No fue sino hasta el aparecimiento de la sociología como ciencia en donde términos como sociedad civil y sociedad política se construyen como conceptos por medio de reconocidos autores clásicos.

Entre los principales se puede mencionar a John Locke y el Segundo tratado del gobierno civil (1689), a Rousseau y el Contrato social (1961) y a Montesquieu con el Espíritu de las leyes (1987). A continuación se detalla, de forma breve, el aporte que cada uno de los autores plantea a la construcción clásica del concepto de sociedad civil.

Locke (1689) define, en primer lugar, una ruptura con el pensamiento de Hobbes al demostrar que bajo ninguna razón un gobierno proviene de alguna fuente divina o su creación se sustenta en algún espacio por fuera de la propia existencia humana. Asimismo, demuestra que el proceso de construcción de la sociedad mantiene una cierta lógica de progreso en cuanto al desarrollo del ser humano desde el estado de naturaleza hasta lo que se denomina por sociedad.

Para Locke la ley de la naturaleza es el espacio en el cual el hombre se encuentra en perfecta libertad y ordena el mundo según juzga su pensamiento; sin embargo, aquel estado no permite, para al autor, el desarrollo pleno del sujeto, por ello transciende a agruparse debido a la existencia de transgresores de la propia ley natural. Para ello plantea la existencia del Estado como el espacio en el cual a ciertos individuos se les concede la capacidad de mantener el orden, la integridad física y a los mismos individuos.

Aunque no surge en su análisis una perspectiva amplia de la sociedad civil, Locke ya sugiere el orden social como el espacio en el cual todos los individuos constituyen un pacto o unión para mantener el orden. Pese a indicar que el Estado mantiene dicha capacidad con la disolución del mismo no implica el comienzo del desorden sino más bien el espacio consentido y visible entre los sujetos que comparten un mismo principio para mantener el estado de naturaleza.

Con Rousseau (1961) se materializa lo que se denomina el mantenimiento de un orden social: "cada uno pone en común su persona y todo su poder bajo la suprema dirección de la voluntad general, y cada miembro considera- 
do como parte indivisible del todo" (Rousseau 1961, 23). Bajo esta premisa, el estado de naturaleza está alejado de un único espacio como la entrega y sumisión de los derechos y deberes de una persona a un gobernante. Pese a buscar siempre el orden social, para Rousseau la prevalencia del mismo se forma mediante la suma de todas las voluntades, el sumar una voluntad y otra conlleva hacia una visión global de construcción del yo común y se mantiene la libertad que cada sujeto mantiene desde el estado de naturaleza.

Este tránsito del estado de naturaleza al estado civil produce en el hombre un cambio muy notable, sustituyendo en su conducta la justicia al instinto y dando a sus acciones la moralidad que antes les faltaba. Sólo entonces es cuando sucediendo la voz del deber al impulso físico y el derecho al apetito, el hombre que hasta aquel momento sólo tenía en cuenta su persona, se ve precisado a obrar según otros principios y a consultar con su razón antes de escuchar sus inclinaciones (Rousseau 1961, 25).

Por lo tanto, el Estado para Rousseau debe ser representativo, pues en el mismo se legitima la sociedad civil. Al ser la autoridad o quien representa al pueblo la suma de las voluntades, es en este espacio que se define el rol de la sociedad civil, pues el hombre pese a entregar su voluntad de igual manera, mantiene su libertad por fuera de quien lo representa. El concepto de sociedad civil en este espacio muestra ser una clara conexión entre el sujeto y el Estado.

Finalmente, Montesquieu (1987) propone el desarrollo de las leyes marcando una clara diferencia entre lo que es el derecho político y el derecho social. Establece como derecho político ${ }^{4}$ al establecimiento de las leyes desde el Estado como ente regulador y propiciador de las mismas, mientras que define al derecho social como la construcción de un tipo de regla que se mantiene bajo el consenso de todos los ciudadanos y permite a la vez una separación entre sociedad civil y Estado.

A finales del siglo XX la academia norteamericana define a la sociedad civil como aquellos grupos sociales que no pertenecen a algún sector económico o menos a la política formal en cuanto se toma como referencia la democracia y los distintos niveles de representación. Con este punto de partida,

4. "Para amar la igualdad y la frugalidad es preciso que las leyes las hayan establecido" (Montesquieu 1987). 
surge dentro del continente americano la necesidad de pensar sobre las organizaciones de la sociedad civil como actores frente a hechos políticos ${ }^{5}$ que las visibilizaron o demostraron su importancia en la construcción del ciudadano.

Se denomina organizaciones de la sociedad civil (OSC), desde la perspectiva teórica de Sergio De Piero (2005), a aquellos grupos de personas que se han organizado como colectivos constituidos y autónomos con el objetivo de no ser meros observadores de los procesos públicos, sino que demandan del Estado y de sus propios agentes la activa participación en la construcción del desarrollo de sus territorios.

Pese a que su conformación se encuentra denominada por distintos valores y/o principios que comparten quienes han decidido formar parte de estos colectivos, De Piero menciona que existe un elemento común denominador entre todas: la acción colectiva como gestora del cambio. Dentro de Occidente, y elemento importante que analiza De Piero debido a la globalización, enfatiza que el denominado tercer sector se ha convertido en el espacio propicio de reproducción y expansión de los regímenes democráticos.

Así, el concepto de sociedad civil se define como "el espacio donde las personas poseen una concepción definida, cercana y no anónima, que favorece la formación de grupos y asociaciones de mutua colaboración, es el espacio de la solidaridad y el intercambio entre personas que se sienten vinculadas por lazos comunes" (Piero 2005, 23).

Una aproximación a lo que se denomina como sociedad civil en el Ecuador, señala Mario Unda como "En Hegel, la sociedad civil era el espacio situado entre la familia y el Estado; la sociedad burguesa en cuanto tal, el lugar en que los intereses particulares se distinguían del Estado" $(2005,48)$.

En Ecuador las organizaciones sociales tienen su origen, ${ }^{6}$ según Unda, en 1972 frente al gobierno revolucionario nacionalista, liderado por militares; época en que el boom petrolero retomó la mirada al Estado como actor benefactor y a la sociedad civil de aquel momento como benefactora del dinero que ingresaba al gobierno. El presente fenómeno materializó los me-

5. Por citar ciertos ejemplos: las primeras organizaciones de trabajadores durante la década del 60 , hasta la década del 80, bajo principios compartidos en donde se buscaba conseguir los derechos laborales en países como Chile, Argentina y Ecuador.

6. Es imposible tener un inicio y un correlato del origen de las organizaciones sociales, el presente documento recoge información secundaria sobre la sociedad civil en Ecuador. 
canismos por los cuales el tercer sector mantendría sus primeras líneas de trabajo; entre las principales el autor menciona: el trabajo con el movimiento indígena en construcción, los sindicatos y las organizaciones de trabajadores, el fortalecimiento al aparato estatal y la beneficencia.

Un segundo momento, menciona el autor, es el retorno a la democracia en 1976, en donde el aparato estatal es criticado y su funcionalidad se pone en duda desde la propia visión del ciudadano que se organiza y protesta; elementos como el alza de pasajes, el ordenamiento territorial en la Costa y la implementación y creación de nuevos partidos, así como de promulgación de nuevas leyes, se convierten en vínculos comunes entre los ecuatorianos que encuentran en la organización y el mantener el conflicto con el Estado una nueva etapa de sociedad civil moderna (Unda 2005).

Un tercer momento es el arranque de la etapa democrática en el país, en donde factores como: la creación de partidos de izquierda, movimientos indígenas y estudiantiles aparecen como espacio de intermediación entre el ciudadano y la sociedad política. Además, menciona el autor, un factor determinante para la aparición formal de las organizaciones de la sociedad civil fue la propia creación y regulación de dichas entidades mediante el Ministerio de Bienestar Social (Unda 2005). Al mismo tiempo y, pese a los primeros acercamientos de representantes políticos y las organizaciones, se mantiene la protesta como eje dominante de los movimientos, así como la influencia de la izquierda como filosofía política del momento.

Un cuarto momento es en el cual se suprimen los modelos cepalinos como referentes hacia el desarrollo y se plantea la libertad de mercado y el inicio de políticas neoliberales, eventos que generan inconformidad entre la sociedad civil del momento, pues sobre todo el movimiento obrero se vio afectado, comenzó a protestar y, al mismo tiempo sucedieron los primeros eventos de represión. Un quinto momento que va de la mano por su acercamiento es la conformación de movimientos insurgentes, por lo cual, el Ministerio de Bienestar Social y la policía generan mecanismos de represión y persecución a líderes; sociales, como consecuencia, la participación ciudadana se ignora y se silencia.

Un sexto momento, resultado de gobiernos de derecha, es cuando la sociedad civil organizada encuentra su espacio propicio de crecimiento y perpetuación en las actividades sobre todo de beneficencia. La iglesia así 
como otras instituciones fortalecen al movimiento social, se plantean programas nacionales de alfabetización, vivienda, acceso a servicios básicos y generación de líderes en territorios rurales (Unda 2005). Sin embargo, este momento es sujeto a deconstrucción al estar en codependencia del Estado y otras instituciones estatales.

Para 1992, y en adelante con el mantenimiento de políticas neoliberales y el incremento del apoyo internacional, las organizaciones sociales crecen en número y personas, se determina a las ONG como sociedad civil dentro del Estado (Unda 2005) y se visualiza su trabajo hacia los ciudadanos. Finalmente a 2005, el autor señala que la sociedad civil busca generar acciones concretas en la construcción de política pública en cuanto a institucionalizar la participación y los derechos.

A partir de 2005, con la proclamación y creación de nuevos partidos, el escenario político y por ende las organizaciones sociales se fragmentaron en cuanto se cierra un ciclo de relación entre Estado y sociedad civil, puesto que los mecanismos como la protesta y participación activa no generaron un nuevo escenario de la realidad social ecuatoriana.

\section{¿Para qué las organizaciones de la sociedad civil en Ecuador?}

Las organizaciones de la sociedad civil en Ecuador han trabajado en su propia construcción como discurso al implantar sus raíces en el movimiento social y la cooperación internacional. De esta manera, han logrado crear un nuevo discurso analítico de lo social (Borja 2011) demostrando así el poder de las ideas.

Al mismo tiempo, al estar inmersas en la lucha social y la reivindicación de derechos, el tercer sector se ha conformado como el ojo crítico de los últimos gobiernos desde el regreso a la democracia.

Para Unda (2005) la construcción de un concepto de sociedad civil en Ecuador implica una ambigüedad en las palabras. Debido a sus usos sociales y conceptuales en nuestro contexto, las organizaciones sociales se encuentran en el barrio, desde la cancha de fútbol en la cual la liga barrial ya representa un nivel de organización de los ciudadanos con valores compartidos; 
frente a una fundación y/o organización sin fines de lucro organizada que refiera su título al ser reconocida por el Estado.

El autor plantea que lejos de pretender dividir a la sociedad civil por su nivel de organización, que siempre va a ser ambiguo, más bien vale proponer un analisis de las intermediaciones que esta realiza con los otros sectores de la sociedad. Detalla que la concepción que tengan tanto Hegel, Marx, Foucault o Gramsci sobre las organizaciones de la sociedad civil sientan, sin lugar a duda, un precedente conceptual; pero para quien las analice es trascendental examinar el espacio de intermediación y vínculo que genera entre el Estado y la sociedad. "El tercer sector no es opuesto al Estado" (Unda 2005, 49). La relación entre Estado y sociedad civil determina, para Unda, la cristalización de las relaciones entre la sociedad política y la sociedad civil.

El tercer sector en el Ecuador es una especie de intersección (Unda 2005) ya que se acerca a la sociedad política en cuanto busca reconocimiento y propone solución a las demandas sociales, tema que es muy difícil, sustenta el autor, ya que el buscar el reconocimiento estatal o social en muchos de los casos carece de apoyo, por tanto representa dinámicas sociales que, por su contexto, notoriamente cambian con el comportamiento y la reproducción social de la vida.

Manifiesta que venir del Estado a la sociedad civil es un punto de confluencia más sencilla, en tanto que la sociedad política requiere un mediador entre la sociedad y ella misma. Por lo tanto, la práctica común del Estado de promover la organización social es eficaz en cuanto le sirva como soporte social frente al resto de actores. Para el autor, esta práctica es un común denominador de todos los gobiernos desde el retorno a la democracia en el Ecuador, por lo que mantiene la duda de si una organización social nace por la necesidad y valores compartidos o por el requerimiento estatal de contar con una sociedad civil acoplada al sistema de dominación (Unda 2005).

\section{Revolución Ciudadana, Estado y sociedad civil: ¿un escenario posible en Ecuador?}

Bajo la propuesta política de establecer un modelo democrático, participativo y revolucionario, efecto de rupturas políticas desde el ámbito inter- 
nacional y sobre todo latinoamericano, bajo la construcción de una nueva izquierda, el texto La nueva política en América Latina: rupturas y continuidades (2008), señala una transformación política de los países de América Latina como proceso de construcción de una nueva izquierda como movimiento político que representaría al ciudadano bajo el nuevo contexto mundial.

La denominación "nuevo" no refiere a un simple cambio electoral sino a un denominador común político en la región caracterizado por una relativamente alta oposición al consenso político reformista de los 90 , la adscripción a ideas y programas políticos tendientes a recompensar algunos de los más agudos efectos sociales, políticos e institucionales (Moreira, Raus y Gómez Leyton 2008, 7).

De esta manera, se presenta en distintos países una nueva figura política comprometida con el cambio social y partidos políticos que recogen una dimensión ideológica con principios como la participación y el desarrollo sostenible de los pueblos. Al mismo tiempo, se establece la necesidad de contar con un nuevo aparataje administrativo-político indispensable en la construcción del Estado moderno desde la demanda de un mundo globalizado.

Sobre estos principios, en Ecuador surge un nuevo movimiento político instaurado por Rafael Correa en 2005, como ministro de Economía en la presidencia de Alfredo Palacio, y nuevas figuras ${ }^{7}$ políticas del contexto ecuatoriano, las mismas que surgen del denominado círculo de economistas progresistas (Moreira, Raus y Gómez Leyton 2008) que postulaban un rol más efectivo del Estado en la economía y la redistribución de la riqueza.

Un escenario político marcado por el descontento social por los partidos políticos que conformaban el Congreso Nacional, errores de negociación del poder político liderado por Alfredo Palacio y su gabinete y la necesidad social de una reforma política, presentaron el contexto ideal para, en las elecciones de octubre de 2006, plantear un cambio político radical con la construcción del Movimiento Alianza País, nuevo movimiento ciudadano, ajeno a toda estructura partidista y comprometido a generar el cambio desde la fundación de un nuevo Ecuador a partir de la conformación de una Asam-

7. En términos generales se trataba de gente vinculada a diversas corrientes de la izquierda (Moreira, Raus y Gómez Leyton 2008). 
blea Constituyente para redactar una nueva Constitución (Moreira, Raus y Gómez Leyton 2008).

Una vez ganadas las elecciones, Rafael Correa con un porcentaje de votos alcanzados en segunda vuelta que sobrepasaron el 50\% del total de votos a nivel nacional, se instaura una plataforma en la que se integrarían movimientos de izquierda relegados desde la presidencia de León Febres Cordero, en conjunto con compañeros y pensadores que acompañaron a Rafael Correa desde sus primeros momentos como imagen visible en el escenario político.

El capítulo I del régimen orgánico de creación del Movimiento Alianza País caracteriza al grupo como:

Un movimiento político democrático, revolucionario de izquierda, alfarista y bolivariano de ciudadanas y ciudadanos que luchan por la Democracia, la igualdad, la equidad, la soberanía, y la autodeterminación de los pueblos, la solidaridad, la justicia social para eliminar la opresión, la dominación, la injusticia y la miseria; con el objetivo de construir el socialismo del Buen Vivir (Movimiento Alianza País 2015, 277).

La Revolución Ciudadana mantiene 13 principios desde su constitución en 2012 como partido político: participación, autonomía respecto al Estado, democracia, transparencia y rendición de cuentas, organización de la sociedad, primacía del interés colectivo sobre el individual, respeto a la diversidad, preocupación y autoformación de los miembros, no violencia, liderazgo colectivo, sentido colectivo, solidaridad y reciprocidad.

\section{El escenario para las organizaciones de la sociedad civil durante la presidencia de Rafael Correa (2007-2017)}

Una vez que se analiza el rol de las organizaciones de la sociedad civil en el Ecuador desde su origen y las principales actividades que cumplen en el país en el presente análisis, es importante determinar su escenario frente al Estado, exactamente desde el primer período de Rafael Correa como presidente constitucional en 2006. Por tal motivo, se recopila información jurídica y de dominio estatal que emergió y recogió lo que para la Revolución Ciudadana significó la sociedad civil en Ecuador. 
En primer lugar, se determina al Estado ecuatoriano como garantista de derechos: la Constitución 2008 en el capítulo II sobre Derechos Civiles ${ }^{8}$ manifiesta que el Estado reconocerá entre otros, el derecho a la libre asociación. El artículo 19 señala:

la libertad de asociación y de reunión. El presente derecho se guía, además, por los tratados internacionales que Ecuador ha suscrito, entre los cuales se encuentran: la Declaración Universal de Derechos Humano y el Pacto Internacional de Derechos Civiles y Políticos (EC 2008).

Existe un marco jurídico que establece la regulación del derecho de asociación en Ecuador, se denomina Código Civil y son normas con carácter de ley. El Estado ecuatoriano denomina como personas no jurídicas a organizaciones de la sociedad civil dentro del territorio, que se muestran como fundaciones o corporaciones, que nacen de las iniciativas ciudadanas con objetivos sociales y sin fines de lucro. Las mismas son objeto de derecho privado y existen una vez que el presidente de la República apruebe su existencia; dicha competencia es delegada a los distintos ministerios en cuanto se alinea la actividad de la organización con las instituciones estatales. Para citar un ejemplo, una organización que trabaja en la promoción del cuidado y protección del medioambiente, recurrirá al Ministerio de Ambiente para que se le otorgue o no, mediante resolución, aprobación.

En segundo lugar, la Ley Orgánica de Participación Ciudadana que nace de la Constitución de 2008 y define a la participación en el artículo 95 como

el derecho de la ciudadanía a participar en todos los asuntos de interés público y que de forma individual o colectiva sean sujetos protagónicos en la toma de decisiones tanto de los asuntos públicos, del control de las instituciones estatales y sus autoridades, así como del trabajo colectivo en favor del bien común; establece la participación como un derecho e institucionaliza la dinámica en torno a la estructura estatal.

Bajo la Ley Orgánica antes mencionada, el Estado pretende garantizar los derechos de las organizaciones de la sociedad civil mediante la creación de un Consejo de Participación Ciudadana y Control Social, y bajo princi-

8. Se denominan como derechos individuales que el ciudadano posee para actuar en la vida pública (Constitución de la República del Ecuador 2008). 
pios como: la promoción a la asociación, promoción estatal a las organizaciones, fortalecimiento a las organizaciones sociales, cogestión de proyectos entre el Estado y la sociedad civil, legalización y registro de las organizaciones sociales y acciones para el voluntariado, la acción social y el desarrollo.

En tercer lugar, se promulga dos decretos ejecutivos (Fundamedios 2017) por parte del presidente Rafael Correa, el primero el 4 junio del 2013, denominado Reglamento para el Funcionamiento del Sistema Unificado de Información de las Organizaciones Sociales y Ciudadanas, que plantea entre sus principales características la definición de sociedad civil como el conjunto de formas organizativas de la sociedad en las que se nombran a: corporaciones, fundaciones, organizaciones extranjeras y organizaciones de control social; sus actividades y promoción estarán guiadas bajo el sistema unificado de información de las organizaciones sociales (RUOS) con el objetivo de regular la existencia, reconocimiento, registro de las actividades que realizan, cierre y control de la gestión de las organizaciones bajo la construcción de instrumentos e instituciones estatales que guíen el trabajo de las OSC en el Ecuador.

E1 Decreto Ejecutivo 739 (Fundamedios 2017) se expide el 13 de agosto de 2015 como: codificación y reformas al Decreto Ejecutivo 16, para homologar los requisitos de otorgamiento de personería jurídica a las organizaciones sociales en el Ecuador, así como obtener un certificado de existencia; se suprime de las distintas denominaciones de las organizaciones sociales a aquellas que se han constituido por instituciones o funciones del Estado, sobre todo para el denominado control social; se plantea el fracaso del registro único para organizaciones, desaparece la obligación a las organizaciones a tener un buen gobierno al interno de cada una; se suprime la causal de cierre de las organizaciones por injerencia política, que fue uno de los elementos más conflictivos en el primer decreto.

A partir del proyecto político denominado Revolución Ciudadana, liderado por Rafael Correa a partir de 2006 como presidente del Ecuador, el contexto para las organizaciones sociales ha sido marcado por un gran contenido normativo y jurídico en conjunto con la politización y desprestigio de las organizaciones sociales en el país, a partir del discurso del presidente. Dicha conclusión no es una simple suma de acontecimientos que han marcado un antes y un después en las organizaciones sociales, sino que es la condición 
que asumió el gobierno de Rafael Correa frente a la lucha y participación social histórica de los movimientos sociales.

Para comprender el discurso que asumió el gobierno de Rafael Correa es indispensable regresar al origen propio de la Revolución Ciudadana. El movimiento político surge en respuesta a los innumerables eventos negativos que acompañaron a los gobiernos del Ecuador desde el regreso a la democracia, en donde la corrupción, la ineficiencia del Estado en cuanto a las demandas sociales y la inalcanzable democracia y participación ciudadana, no formaban parte aún del discurso político, menos aún de la política nacional. El pretender recoger las demandas históricas de los movimientos sociales se transformó en estrategia para llegar al poder.

La Revolución Ciudadana aún en construcción y quienes ya formaban parte del movimiento, encontraron en la elaboración del discurso político de la reivindicación social bajo un modelo democrático participativo, el espacio ideal para construir propuestas alineadas a diversos movimientos y organizaciones sociales que mantenían espacio y prestigio dentro de la sociedad ecuatoriana por su lucha y defensa por los derechos, la participación y control a las organizaciones estatales y su trabajo en favor del desarrollo del Ecuador. El establecer una participación en democracia generó en los ciudadanos y movimientos su adscripción en un primer momento, por lo tanto, no es de extrañeza que durante 2005 e inicios de 2006 la Revolución Ciudadana tuvo acercamientos y reuniones con el movimiento indígena y otros actores sociales, con el claro objetivo de validar en cuanto representaban o mantenían el apoyo de los grandes e históricos movimientos sociales. Apoyo o representación que se tradujo en las elecciones de 2006 bajo una propuesta de gobierno dirigida a la sociedad civil en dos acciones concretas: demandas sociales al Estado y mecanismos de participación dentro del escenario a futuro del Ecuador; propuestas que entre otras dieron la victoria a Rafael Correa en segunda vuelta electoral en noviembre de 2016. La Revolución Ciudadana una vez consolidada como movimiento político, estableció la necesidad de contar con una nueva Constitución, una fundación más del Ecuador a partir de un instrumento fundamental para el Estado desde la construcción de la misma, a partir de la participación de todos los sectores de la sociedad ecuatoriana y que fue aprobada vía referéndum por los ecuatorianos 
con un $63,93 \%$, según datos del llamado hasta entonces Tribunal Supremo Electoral.

La Constitución de 2008 estableció, como se menciona al inicio de este análisis, en primer lugar un incremento del aparato estatal con el objetivo de promover y garantizar los distintos e innovadores cambios a todo nivel; en segundo lugar, se institucionalizó la participación y se definió la misma a partir del Estado como ente regulador. Este efecto genera un antes y un después para las organizaciones de la sociedad civil en el Ecuador, ya que históricamente la lucha social, la promoción y defensa de derechos, así como los valores morales que atravesaron el tiempo y espacio y mantuvieron la participación activa de los movimientos sociales, se vio reducida en cuanto se creó una Ley Orgánica de Participación Social que no tomó en cuenta las dinámicas y espacios de la participación.

Se convirtió a la participación en una instrumentalización y creación de normas e instituciones estatales que tienen como principio, hasta el día de hoy, la promulgación y promoción de la participación. Sin embargo, lo que ha ocurrido es todo lo contrario. Contar con todo un marco definido y cerrado de lo que es y cómo se debe generar la participación, generó en el contexto ecuatoriano ciertos fenómenos que son importantes mencionarlos, como: la ruptura de las relaciones de los movimientos sociales, sobre todo de los más visibles como el indígena y el ambiental, con el gobierno; la construcción de un discurso político de ataque a la sociedad civil por parte del presidente Rafael Correa y un contexto marcado por la incertidumbre para las organizaciones debido a un agresivo control a sus actividades y permanente desprestigio como gestores del desarrollo.

El gobierno de Rafael Correa instrumentalizó la participación e hizo de uno de los principios más importantes y compartidos en la sociedad civil una herramienta política con dos objetivos claros: el primero, fortalecer el rol del Estado como rector del proceso de desarrollo del país, lo que significó desvalorar el trabajo de los otros actores dentro del Ecuador; el segundo, es que el Estado asumió el rol como único agente para el cambio social bajo su propia estructura. Estos objetivos permitieron al gobierno ser el centro de atención del ciudadano, se convirtió el Estado en organismo proponente, ejecutor y evaluador del desarrollo social del país; con esto se invalidó el discurso de 
promotores del cambio social por parte de la sociedad civil, así como la inestabilidad y continuidad de sus actividades debido al robustecimiento estatal.

Sin embargo, no son solo los cambios estructurales y normativos un escenario que dificultó el trabajo de las organizaciones de la sociedad civil; se debe sumar además la desarticulación social como práctica del gobierno de Rafael Correa, debido a que en un primer momento y por intereses políticos se asumieron luchas y discursos provenientes de la sociedad civil que solo fueron útiles en cierto momento. Un ejemplo claro es la apropiación del discurso indígena desde finales de 2005 y que terminó con la ruptura y criminalización de sus dirigentes.

Otro factor determinante para concluir que el escenario al cual las organizaciones de la sociedad civil se enfrentaron durante la presidencia de Rafael Correa fue la discrecionalidad de la normativa creada para controlar la sociedad civil ecuatoriana; hasta la actualidad existen más de 322 normas legales vigentes según la Confederación Ecuatoriana de Organizaciones de la Sociedad Civil (CEOSC 2010), institución que regula la constitución y funcionamiento de las organizaciones a partir de evidentes facultades otorgadas al poder ejecutivo. Uno de los mayores y evidentes espacios en los que se refleja el control del Estado es la facultad para el cierre y/o disolución de las organizaciones sociales que se describen en el Decreto 16, sección sexta, en la que el Estado describe entre las principales causas de cierre la injerencia en política. De esta facultad el Estado ya ha hecho uso y se refleja en el cierre de ciertas organizaciones.

En base a información proporcionada por Fundamedios y la CEOSC en sus portales digitales, ya se restringen desde el poder ejecutivo derechos como la libre asociación. Existen casos como el de Acción Ecológica, Fundación Pachamama y la Unión Nacional de Educadores que fueron notificadas de procesos de cierre o incluso de disolución bajo discrecionalidad del poder ejecutivo, acusándolas de haber realizado injerencia política o que se hayan alejado de sus principios como organización.

Información proporcionada por la Secretaría Nacional de Gestión de la Política (2007), institución estatal a la que se le otorgó las funciones de articular el diálogo político como representante del Ejecutivo en territorio, presenta que desde 2007 a 2017 se han registrado un total de 91.043 organizaciones de las cuales 18.348 han sido ya registradas, 72.695 se encuentran 
en proceso de registro; se han liquidado 4 organizaciones y se han disuelto 44. La disolución, desde la información que se puede recoger debido a lo polémico que puede llegar a ser hablar sobre la sociedad civil hoy en día desde quienes son servidores públicos, evidencia cierre por distintas razones como: disminución de miembros o falta de entrega de papeles. Sin embargo, información sobre disolución por injerencia política es casi imposible que sea proporcionada por la institución.

El contexto para las organizaciones de la sociedad civil en el Ecuador se describe bajo la discrecionalidad del poder ejecutivo sobre una dinámica constante y variable como es la de las organizaciones de la sociedad civil por medio de la regulación y control. El principio de la participación al momento de ser instrumento político, elimina su origen y validez en el imaginario colectivo y se transforma en un simple requisito adicional para el Estado y el cumplimiento de sus objetivos. Para la sociedad civil ecuatoriana se vislumbra un escenario de incertidumbre debido a la capacidad estatal en cuanto a normativa e instituciones para generar control, mas no fomentar y valorar las acciones que las organizaciones sociales han realizado como agentes del desarrollo.

Información recolectada de 2010 a 2016 por parte de la CEOSC (2010) en su rendición de cuentas, documento que recopila la información y actividades de las organizaciones con un análisis integral, demuestra que la sociedad civil en Ecuador es un verdadero agente del desarrollo ya que su trabajo está vinculado a actividades tan infinitas como importantes que van desde: cultura, educación, movilidad, participación, democracia, género, ambiente, salud, discapacidades, entre muchas más. Elementos que sin la previa necesidad de identificarlos en un proceso de desarrollo desde una perspectiva teórica colaboran sin duda al progreso de la sociedad ecuatoriana.

Aunque el gobierno ha planteado un plan de desarrollo en el que se evidencia un proceso de construcción colectiva en principio, la realidad y contexto de los actores que se involucran en el desarrollo del país están sujetos a una estructura de control por parte del Poder Ejecutivo, lineamientos disciplinarios de la participación, lo que limita en muchas veces las acciones de la sociedad civil y han generado un contexto hasta el 25 de mayo de 2017 furtivo de la sociedad civil ecuatoriana en base a valores morales compartidos e ideales de construcción desde lo colectivo de un Ecuador diferente. 


\section{A manera de conclusiones}

- Surge la sociedad civil en Ecuador como el grupo de individuos que comparten un valor común, una lucha común; se convierte en bandera de lucha la preservación del ambiente, la promoción y defensa de derechos y la acción colectiva como respuesta a la ya debilitada sociedad política. Con esto, las organizaciones sociales desde la década del 60 hasta el 80 exploran su mayor visibilización y con esto la importancia que tiene en el desarrollo del país.

- Históricamente, la lucha social y la defensa de derechos ha sido la mayor exposición del trabajo de la sociedad civil en Ecuador, es por ello que desde el retorno a la democracia el Estado encontró en los movimientos sociales un respaldo tanto político como de cooperación para buscar soluciones a la problemática social.

- Bajo la propuesta política de una Revolución Ciudadana, el movimiento Alianza País se avista como una nueva figura política comprometida con el cambio social y la participación; además, se plantea un modelo de desarrollo que gira la dinámica nacional al tomar como lineamientos principios como: igualdad, cumplimiento de derechos, reconstrucción de lo público, democracia participativa y representativa y la construcción de un Estado diverso, entre otros.

- Un punto determinante para el movimiento Alianza País y su plan de desarrollo es la participación y construcción del ciudadano. Desde ahí, hasta la Constitución de 2008 y las innumerables normativas que tenían el objetivo de materializar la participación dejaron en la sociedad ecuatoriana, y sobre todo en las organizaciones de la sociedad civil un retroceso, en el proceso de diálogo con la sociedad política.

- Desde el Poder Ejecutivo, en su afán de normar y controlar a la sociedad civil en el Ecuador (primer error debido al desconocimiento de la dinámica de los colectivos sociales), se propuso una ley orgánica de participación social y dos decretos específicos para el funcionamiento de las organizaciones con un solo objetivo: la reificación a la sociedad civil en el país.

- Como movimiento político, Alianza País tomó las luchas de los movimientos sociales y las adaptó a su plan de gobierno, lo que generó la 
desarticulación de las organizaciones sociales y la ilegitimidad en sus acciones, pues con tres períodos en la presidencia de Rafael Correa se multiplicaron y engrosaron las acciones estatales en el bienestar de la sociedad, lo que ocasionó una desestructuración ya no solo a nivel político sino a nivel organizativo de los colectivos.

- El falso sueño de participación alejó del ciudadano las prácticas colectivas, el reconocimiento como actor dentro de la gestión pública y delimitó su accionar a elementos concretos como el sufragio o el apoyo desde una condición más política. Por lo tanto, los movimientos sociales, desde toda una visión macro, como actores se desmantelaron con claros objetivos políticos, ya que quien no era partidario de las acciones gubernamentales o poseía una visión crítica del mismo se sometía al control y ofensa desde el discurso político del movimiento.

- Pese a caracterizarse como un escenario de incertidumbre, las organizaciones de la sociedad civil han buscado espacios y medios para poder continuar sus actividades en un escenario difícil, el mismo que les ha motivado a encontrar un consenso dentro de ellas mismas y es evidente al día de hoy ciertas aproximaciones a buscar en conjunto un ambiente propicio para su desarrollo y su relación con el Estado.

- No basta solo con cambios a nivel de la normativa o el cambio de gobierno para reconocer y valorar las acciones de la sociedad civil en el país. Es indispensable retomar a la conciencia social y la construcción de un Ecuador desde la multiplicidad de actores.

\section{Referencias}

Aristóteles. 1962. La política. Traducido por Natividad Massanés. Barcelona: Iberia.

Borja, Raúl. 2011. Los movimientos sociales en los 80 y 90, la incidencia de las ONG, la iglesia y la izquierda. Quito: Centro de Investigaciones CIUDAD.

Confederación Ecuatoriana de Organizaciones de la Sociedad Civil (CEOSC). 2010. Primer informe de rendición de cuentas. Informe de gestión. Quito: Grupo FARO.

De Piero, Sergio. 2005. Organizaciones de la sociedad civil: tensiones de una agenda en construcción. Buenos Aires: Paidós SAICF.

Ecuador. 2008. Constitución de la República del Ecuador. Registro Oficial 449, 20 de octubre. ---. 2008. Ley Orgánica de Participación Ciudadana. Registro Oficial 175, 20 de abril. 
---. 2008. Código Civil Ecuatoriano. Registro Oficial 46, 24 de junio.

---. 2015. Decreto Ejecutivo 739. Registro Oficial 570, 21 de agosto. Reglamento para el Funcionamiento del Sistema Unificado de Información de las Organizaciones Sociales y Ciudadanas.

Fundamedios. 2017. "Los decretos 16 y 739 ¿simples mecanismos para disolver organizaciones?". Fundamedios. Consulta: mayo de 2017. «ttp://www.fundamedios.org/tag/decreto->.

Locke, John. 1689. Segundo tratado sobre el gobierno civil: un ensayo acerca del verdadero origen, alcance del gobierno civil. Madrid: Tecnos.

Montesquieu, barón de (Charles de Secondat). 1987. El espíritu de las leyes. Madrid: Tecnos.

Moreira, Carlos, Diego Raus y Juan C. Gómez Leyton, coords. 2008. La nueva política en América Latina: rupturas y continuidades. FLACSO-Sede Uruguay. Montevideo: Trilse.

Movimiento Alianza País. 2015. "Capítulo I del régimen orgánico de creación del Movimiento Alianza País". Alianza País. Consulta: agosto de 2017. 〈https://www.alianzapais.com. ec/2015/07/03/regimen-organico/s.

Real Academia Española. 2017. Consulta: agosto de 2017. «https://dle.rae.es/〉.

Rousseau, Juan Jacobo. 1961. El contrato social o principios del derecho político. Buenos Aires: Compañía General Fabril Editora.

Secretaría Nacional de Gestión de la Política. 2007. Consulta: agosto de 2017. «https://www. politica.gob.ec/s.

Touraine, Alain. 2000. ¿Podremos vivir juntos? La discusión pendiente: el destino del hombre en la aldea global. Buenos Aires: Fondo de Cultura Económica.

Unda, Mario. 2005. Luces y sombras: voces y miradas sobre la sociedad civil de hoy. Quito: Centro de Investigaciones CIUDAD. 